\title{
A Numerical Simulation of Deepwater Riser's FSI based on MLPG
}

\author{
Jianping Chen ${ }^{\mathrm{a}, *}$, Jie Xu ${ }^{\mathrm{b}}$, Litao Wang ${ }^{\mathrm{a}}$, Xinen Chen ${ }^{\mathrm{a}}$, You Gong ${ }^{\mathrm{a}}$ \\ ${ }^{a}$ School of Ship Engineering, Guangzhou Maritime University, Guangzhou, 510725, China \\ ${ }^{b}$ Faculty of Automation, Guangdong University of Technology, Guangzhou, 510006, China
}

\begin{abstract}
The paper presents the numerical simulation technology of deep-water risers' fluid-solid interaction based on mesh-free MLPG method, which applies the combination of Petrov-Galerkin Method and three-step finite element method in the mode of separating velocity and pressure to discretize control equation of two-dimensional incompressible flow field respectively in time and space. At the end of the paper, a stationary cylinder Vortex-Induced Vibration is taken as an example to be calculated and compared. It is in good agreement with the traditional numerical method based on mesh and physical model well, which proves the proposed method is effective and has good accuracy.
\end{abstract}

Keywords: deep-water riser; vortex-induced vibration; fluid-solid interaction; numerical simulation; mesh-free method

(Submitted on October 17, 2017; Revised on December 3, 2017; Accepted on January 22, 2018)

(C) 2018 Totem Publisher, Inc. All rights reserved.

\section{Introduction}

Riser is an important equipment for Deepwater oil and gas resources development [18]. With the effect of incoming flow, there will be alternate vortexes shedding in both sides of the risers. When the vortex shedding frequency is close to the natural one, the phenomenon of Vortex-induced Vibration (VIV) occurs, which engenders vortex-induced lift forces and drag forces on risers. Thus, risers' fatigue failure occurs [8].

VIV, a typical nonlinear Fluid-solid Interaction (FSI) problem, is also one of the key problems of risers' study and design. Back in 1979, Williamson and Roshko proposed two oscillation modes in the frequency "Lock-in" area, called 2S, 2P mode. In the follow-up work, supported by Govardhan and Khalak, they studied VIV frequency response and damping effects of elastic cylinder with low mass from 1990 to 2002 through a series of experiments [4,5,9]. Meanwhile, some scholars also carried out VIV suppression device experimental study. The numerical simulation of VIV is also one of the hot studies. Though the deep-sea riser's FSI theory and research began late in our country, some achievements had been made $[2,11,14,17]$. For instance, considering the cross-flow's effect on forward effect, Tang Wenyong, Xue Hongxiang and et al established deep-sea risers forward flow forecasting mode [15]. Wang Yifei, Cui Weicheng and et al made use of the VIV forecasting program to calculate risers' motivation response in different parameters according to the flow speed distribution along depth direction in South China Sea, Gulf of Mexico and West Africa [16]. Chen Weiming, Li Min and et al studied the big length to diameter ratio risers' frequency locking properties acted by internal wave flow field, and established calculation based on multi-mode frequency locking of modal energy theory [3]. Yue Qianjin, Lei Song and et al applied fourth-order Runge-Kutta to solve the mentioned nonlinear equation and analyzed the FPSO heaving movement's effect on VIV [10].

Based on the experimental data of the oscillated cylinder and energy balance principle forecasting the VIV response and the induced fatigue damage of the riser, Tang Wenyong, Xue Hongxiang, Zhang Xiaoying and et al applied surface method to be established the safety factor of VIV fatigue and the limit state equation. The influence of fatigue safety factor on structural reliability is studied, and the sensitivity of each random variable is analyzed [19].

\footnotetext{
* Corresponding author.

E-mail address: jianpingchenjpc@ sina.com
} 
In the CFD method, such as VIV, the typical computational process is based on the Navier-Stokes (N-S) equation, while the finite-element method (FEM) is usually used to discretize the governing equations. In the Deepwater riser VIV calculation, the riser is slender and easy to produce great deformation, so Deepwater riser VIV has a strong nonlinear. When using the FEM method to analyze nonlinear problems, there will be some units of poor quality; some units may become very sharp, thereby reducing the accuracy of the calculation. In the field of computational fluid mechanics, free of meshes is the most attractive feature of meshless methods. In 1997, Belytsch and et al proposed a three-dimensional explicit EFG method and used this method to simulate the sloshing of non-viscous fluid in a sink [1]. In 2000, Do and et al applied the meshless method to analyze the shallow water flow problem in two-dimensional steady-state rivers [3]. By applying SUPG stabilization technique to the Galerkin equation, the effects of convection terms are considered, and the effects of the number of basic functions and the local support domain of the nodes on the computational time and computational accuracy of the EFG method are studied in detail. In 2005, Singh presented a parallel algorithm for EFG [13]. Based on the data decomposition, it can solve problems when the coefficient matrix formed by the EFG method is a sparse matrix and a nondefinite matrix and that the computation is large. Based on this parallel algorithm, the viscous incompressible flow (Stokes flow) and the two-dimensional heat transfer problem were solved by four-processor workstation parallel computation, and the calculation efficiency and accuracy were better. In the same year, Singh also used the parallel computation method to calculate the three-dimensional heat conduction problem and validated the effectiveness of the parallel algorithm for the EFG method. At present, the computational complexity of EFG method is still a little high (higher than that of FEM method), and the treatment of essential boundary conditions is still a little complex. Therefore, the theoretical and computational methods need to be improved. In the field of nonlinear FSI analysis, there has been no research on meshless methods at home and abroad.

In this paper, the Vortex-Induced Vibration of riser is taken as the research background. The application of 2-dimension fixed cylinder VIV is used as an example to analyze and discuss the application of 2-D fluid-structure interaction in numerical simulation, and to discuss the calculation effect and calculation precision of this method compared with the traditional grid-based numerical method and the foreign physical model experiment. First, based on Meshless Local PetrovGalerkin (Meshless Local Petrov-Galerkin, MLPG) method, it applies the combination of MLPG and three-step finite element method in the mode of separating speed and pressure to discretize governing equation of flow field by the EFG method in space and by three-step finite element method in time. Take the two-dimensional fixed cylinder VIV as an example; the flow pattern and the relevant VIV parameters were analyzed by the above method to calculate the force of the flow field and the lift coefficient $C_{l}$, the drag coefficient $C_{d}$ and the Strouhal number $S t$ of different nodes, which can verify the method's efficiency to the above problem.

\section{Basic expression of MLPG method for2-D flow}

Based on the 2-D elastic theory, the nodal system equations after flow field is discretized on the basis of two-dimension as Equation (1)

$$
\sigma_{i j, i}+b_{i}=0
$$

Where $\Omega$ is defined as problem domain, $\Gamma$ as boundary of $\Omega$. The essential boundary conditions of the problem are $u_{i}=\bar{u}_{i}, \Gamma_{u}$. The natural boundary condition of the problem is $\sigma_{i j} n_{j}=t_{j}, \Gamma_{t}, n_{j}$ is the $j^{t h}$ component of outer normal vector on natural boundary.

Using the weighted residual method, we can get the strong differential equation of the system equation at the node $I$.

$$
\int_{\Omega_{Q}}\left(\sigma_{i j, i}+b_{i}\right) W_{I} d \Omega-a \int_{\Gamma_{Q_{u}}}\left(u_{i}-\bar{u}_{i}\right) W_{I} d \Gamma=0
$$

Where $W_{I}$ is the weight function, $\Omega_{Q}$ is the integral domain of Node $I, \Gamma_{Q}$ is its boundary, and $\Gamma_{Q_{u}}$ is the coincidence part of the Integral Domain $\Omega_{Q}$ and the Essential Boundary $\Gamma_{u}, \alpha$ is the penalty factor.

According to Equation (2), integrating Node $I$ in its integral domain, the system equation can be established. 
Therefore, all discretized nodes can be established in this way. And if we assemble these equations, we can obtain the overall system equations of the problem domain.

The filed function of node's' integral support can be obtained by using Method of Least Squares (MLS), which makes the displacement of the problem domain close to the function as Equation (3).

$$
u^{h}(X)=\Phi^{T}(X) \cdot u=\sum_{I=1}^{N} \phi_{I}(X) u_{I}
$$

Where $\Phi^{T}(X)$ is the field function matrix obtained by MLS, $u_{I}$ is value of discretized Node $I$, and $N$ is the node number of integral's support domain $\Omega_{\mathrm{s}}$.

Set $V_{I}$ as the derivative coefficient matrix of weight function $W_{I}$, so Equation (2) can be expressed in a further way as following.

$$
\begin{aligned}
\int_{\Omega_{Q}} V_{I} \sigma d \Omega & +\alpha \int_{\Gamma_{Q_{u}}} W_{I} u d \Gamma-\int_{\Gamma_{Q_{u}}} W_{I} N \sigma d \Gamma \\
& =\int_{\Gamma_{Q_{t}}} W_{I} \bar{t} d \Gamma+\alpha \int_{\Gamma_{Q_{u}}} W_{I} \bar{u} d \Gamma+\int_{\Omega_{Q}} W_{I} b d \Omega
\end{aligned}
$$

Where $\Gamma_{Q_{t}}$ is the coincidence part of the natural boundary and integral domain $\Omega_{Q}, \quad N$ is component of outer normal vector matrix on natural boundary as Equation (4).

\section{The Flow Field's control Equation and Discretization}

For deep-sea risers, we can perform slice analysis to simplify the analyzing procedure. Every slice can be regarded as a $2 \mathrm{D}$ problem. For numerical analysis of 2D FSI problem, its priority is to calculate flow field's acting force according to CFD. The control equation of two-dimensional incompressible fluid:

$$
\left\{\begin{array}{l}
\frac{\partial u_{i}}{\partial t}+u_{j} u_{i, j}=-\frac{p_{, i}}{\rho}+v\left(u_{i, j}+u_{j, i}\right)_{, j}+f_{i} \\
u_{i, i}=0
\end{array}\right.
$$

Where, $f_{i}$ is body force, $u_{i}$ is flow velocity, $v$ is the kinematic viscosity of fluid as Equation (5).

The essential boundary condition of flow filed is Equation (6)

$$
\left\{\begin{array}{l}
u_{i}=u_{i} \\
\sigma_{i j}=-\frac{p \delta_{i j}}{\rho}+v\left(u_{i, j}+u_{j, i}\right)=\sigma_{i j}
\end{array}\right.
$$

Where $\sigma_{i j}$ is stress, $u_{i}$ and $\sigma_{i j}$ are respectively the known flow speed and stress.

We can apply the mode of separating speed and pressure to discretize the control equation of fluid in time and space respectively, employing three-step finite element method in time and MLPG method in space. 
Based on three-step finite element method, the sub-step radical of velocity component in time is:

$$
\left\{\begin{array}{l}
u_{i}^{n+1 / 3}=u_{i}^{n}+\frac{\Delta t}{3} \frac{\partial u_{i}^{n}}{\partial t} \\
u_{i}^{n+1 / 2}=u_{i}^{u}+\frac{\Delta t}{2} \frac{\partial u_{i}^{n+1 / 3}}{\partial t} \\
u_{i}^{n+1}=u_{i}^{u}+\Delta t \frac{\partial u_{i}^{n+1 / 2}}{\partial t}
\end{array}\right.
$$

In Equation (7), the mode of velocity separating in time is close to Taylor's expansion of the third-order accuracy term. According to Equation (9-11), time discretizing of N-S equation can be manifested as following.

$$
\left\{\begin{array}{l}
\frac{u_{i}^{n+1 / 3}-u_{i}^{n}}{\Delta t / 3}=-u_{j}^{n} u_{i, j}^{n}-\frac{p_{, i}^{n}}{\rho}+v\left(u_{i, j}^{n}+u_{j, i}^{n}\right)_{, j}+f_{i}^{n} \\
\frac{u_{i}^{n+1 / 2}-u_{i}^{n}}{\Delta t / 3}=-u_{j}^{n+1 / 3} u_{i, j}^{n+1 / 3}-\frac{p_{, i}^{n+1 / 2}}{\rho}+v\left(u_{i, j}^{n+1 / 3}+u_{j, i}^{n+1 / 3}\right)_{, j}+f_{i}^{n+1 / 3} \\
\frac{u_{i}^{n+1}-u_{i}^{n}}{\Delta t / 3}=-u_{j}^{n+1 / 2} u_{i, j}^{n+1 / 2}-\frac{p_{, i}^{n+1}}{\rho}+v\left(u_{i, j}^{n+1 / 2}+u_{j, i}^{n+1 / 2}\right)_{, j}+f_{i}^{n+1 / 2}
\end{array}\right.
$$

By using MLPG method to discretize control equation of flow field in space and using Lagrange Multiplier Method to solve the essential boundary condition, the weak form of the control equation can be obtained.

$$
\int_{\Omega}(L \delta u)^{T}(D L u) d \Omega=\int_{\Omega} \delta u^{T} b d \Omega+\int_{\Gamma_{t}} \delta u^{T} \bar{t} d \Gamma+\int_{\Gamma_{u}} \delta \lambda^{T}(u-\bar{u}) d \Gamma+\int_{\Gamma_{u}} \delta u^{T} \lambda d \Gamma
$$

Regarding Lagrange Multiplier $\lambda$ as location function of coordinate as well, the nodal values and shape functions of the essential boundary conditions are interpolated, and we can obtain the following.

$$
\lambda=\sum_{I}^{n_{\lambda}}\left[\begin{array}{cc}
N_{I} & 0 \\
0 & N_{I}
\end{array}\right]\left\{\begin{array}{c}
\lambda_{u_{I}} \\
\lambda_{v_{I}}
\end{array}\right\}=\sum_{I}^{n} N_{I} \lambda_{I}
$$

Where $N_{I}$ is the shape function matrix of node $I$ on essential boundary.

First order Lagrange interpolation function (linear interpolation function) is used to simplify the calculation in this paper; we can obtain the discretized equation as following. 


$$
\left\{\begin{array}{l}
M_{\alpha \beta} \frac{u_{i \beta}^{n+1 / 3}-u_{i \beta}^{n}}{\Delta t / 3}= \\
\quad-N_{\alpha \beta}^{n} u_{i \beta}^{n}-\frac{L_{i \alpha \alpha} \rho_{\beta}^{n}}{\rho}-v S_{i \alpha}^{n}+M_{\alpha \beta} f_{i \beta}^{n}+\int_{\Gamma} \Phi_{\alpha} v\left(u_{i, j}^{n}+u_{j, i}^{n}\right) n_{i} d \Gamma \\
M_{\alpha \beta} \frac{u_{i \beta}^{n+1 / 2}-u_{i \beta}^{n}}{\Delta t / 2}= \\
\quad-N_{\alpha \beta}^{n+1 / 3} u_{i \beta}^{n+1 / 3}-\frac{L_{i \alpha \alpha} \rho_{\beta}^{n}}{\rho}-v S_{i \alpha}^{n+1 / 3}+M_{\alpha \beta} f_{i \beta}^{n+1 / 3}+\int_{\Gamma} \Phi_{\alpha} v\left(u_{i, j}^{n+1 / 3}+u_{j, i}^{n+1 / 3}\right) n_{i} d \Gamma \\
M_{\alpha \beta} \frac{u_{i \beta}^{n+1}-u_{i \beta}^{n}}{\Delta t / 3}= \\
\quad-N_{\alpha \beta}^{n+1 / 2} u_{i \beta}^{n+1 / 2}-\frac{L_{i \alpha \alpha} \rho_{\beta}^{n+1}}{\rho}-v S_{i \alpha}^{n+1 / 2}+M_{\alpha \beta} f_{i \beta}^{n+1 / 2}+\int_{\Gamma} \Phi_{\alpha} v\left(u_{i, j}^{n+1 / 2}+u_{j, i}^{n+1 / 2}\right) n_{i} d \Gamma
\end{array}\right.
$$

Where

$$
\begin{aligned}
& M_{\alpha \beta}=\iint_{\Omega^{e}} \Phi_{\alpha} \Phi_{\beta} \\
& L_{i \alpha \beta}=\iint_{\Omega^{e}} \Phi_{\alpha} \Phi_{\beta, i} d \Omega \\
& N_{\alpha \beta}^{n}=\iint_{\Omega^{e}} \Phi_{\alpha} \Phi_{\nu} \Phi_{\beta, j} u_{j v}^{n} d \Omega \\
& S_{i \alpha}^{n}=\iint_{\Omega^{e}} \Phi_{\alpha, i}\left(\Phi_{\beta, j} u_{i \beta}^{n}+\Phi_{\beta, i} u_{j \beta}^{n}\right) d \Omega
\end{aligned}
$$

According to Equation (7-11), $p^{n+1}$ have to be obtained first if we want to get node displacement $u_{i}^{n+1}$ 。For the third equation in Equation (8), we can take divergence in both side and introduce incompressible boundary condition $u_{i, i}^{n+1}=0$ to get Poisson equation about pressure.

$$
\frac{p_{, i i}^{n+1}}{\rho}=\frac{u_{i, i}^{n}}{\Delta t}-\left(u_{j}^{n+1 / 2} u_{i, j}^{n+1 / 2}\right)_{, i}+v\left(u_{i, j}^{n+1 / 2}+u_{j, i}^{n+1 / 2}\right)_{, j i}+f_{i, i}^{n+1 / 2}
$$

Discretize Equation (12) by MLPG and finally the pressure used equation of FSI meshless numerical analysis can be obtained.

$$
G_{\alpha \beta} p_{\beta}^{n+1}=-\frac{1}{\Delta t} H_{i \alpha \beta} u_{i \beta}^{n}-K_{j \alpha \beta} u_{j \beta}^{n+1 / 2}-S_{\alpha}
$$

Where

$$
\begin{aligned}
& G_{\alpha \beta}=\iint_{\Omega^{e}} \Phi_{\alpha, i} \Phi_{\beta, i} d \Omega \\
& H_{i \alpha \beta}=\iint_{\Omega^{e}} \Phi_{\alpha} \Phi_{\beta, i} d \Omega \\
& K_{j \alpha \beta}=\iint_{\Omega^{e}} \Phi_{\alpha, i} \Phi_{\beta} \Phi_{v, j} u_{i v}^{n+1 / 2} d \Omega \\
& S_{\alpha}=\int_{\Gamma} \Phi_{\alpha} \frac{u_{i}^{n+1}-u_{i}^{n}}{\Delta t} d \Gamma
\end{aligned}
$$

Therefore, according to Equation (13), $p^{n+1}$ can be obtained, and $u_{i}^{n+1}$ can be obtained by Equation (12). 


\section{The Iteration Algorithm of Interaction Motion}

The structure's motion in flow filed is caused by fluid, but its motion is not the same of that of the fluid. In numerical calculation, we generally postpone a time step of the structure's motion. Therefore, if we choose a shorter step, the accuracy of calculation will be better and the calculate complexity will be increased. According to relevant references, we use the physical time step to $0.002 \mathrm{~s}$ to calculate and obtain the corresponding dimensionless step by an example.

The basic thought of algorithm is listed as follows. First, solve lift force $F_{L}$ and drag force $F_{D}$ according to the velocity filed and pressure filed obtained above. Then, solve its equation of motion, and obtain the structure's motion response. Pass the motion velocity to the node of the interaction interface, and calculate the node's displacement by the processing algorithm of moving boundary node. When the node iteration calculation has been converged, regard it as the new boundary condition to calculate the time step flow filed. Cycle the iteration this way until the converged numerical value. In the calculation of flow field, structure motion equation and the processing of moving boundary node, we adapt separated block and the mode of file exchange to deliver data.

The algorithm in this paper proposes node adaptive discretizing method according to the special requirement of node discretizing. First, post specific amount of discretizing node on the structure surface boundary and flow field boundary. Then, according to given background mash information, choose a node randomly and calculate its effect radius in the calculate domain. Last, circle with the effect radius as local support domain to analyze the node's circumstance in the local support domain (or its sub-domain). Finally, create new nodes automatically according to the spacing requirement of node.

\section{Numerical example}

\subsection{Calculation area and condition}

In the paper, a sliced 2D simulating deep-water riser is taken as an example to testify the effectiveness and accuracy of numerical simulation method for fluid - solid interaction. In order to simplify the calculation and to facilitate analysis, the following example is used as shown in Figure 1.

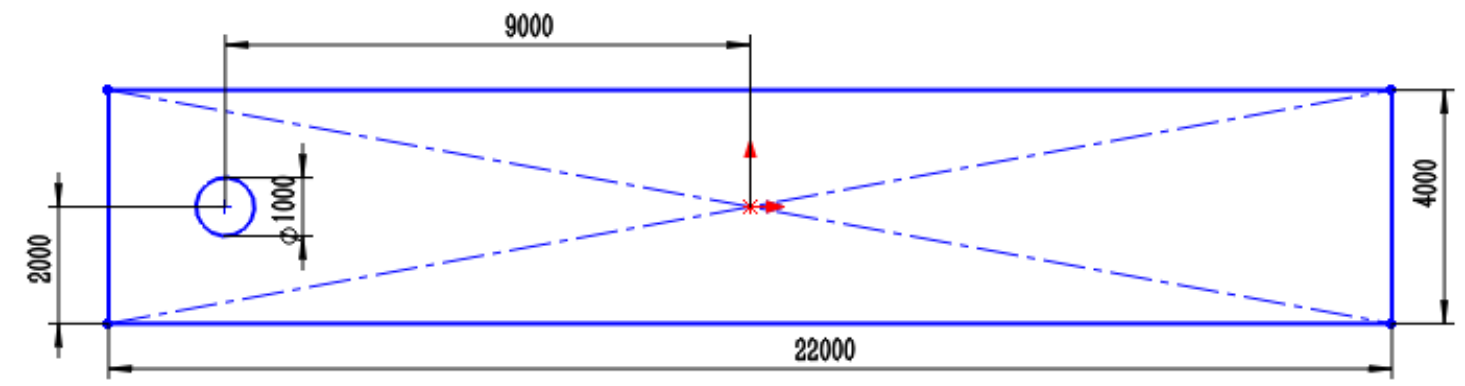

Figure 1. Computation region of two-dimensional incompressible flow field

Cylinder diameter $D=1.0 \mathrm{~m}$. The flow velocity is distributed according to Equation (14)

$$
\left\{\begin{array}{l}
u_{x}=\frac{6 U}{H^{2}}\left[\left(y-y_{B}\right) H-\left(y-y_{B}\right)^{2}\right] \\
u_{y}=0
\end{array}\right.
$$

Where, the average flow velocity is $U=1.0 \mathrm{~m} / \mathrm{s}, \quad H$ is the height of the flow field used in the calculation.

\subsection{Calculate the layout algorithm used}

In the example, auto-layout method is taken to arrange nodes. First, in the cylinder's surface and flow field boundary, arrange a specific amount of initial nodes in the flow field. Then, concentrate nodes automatically according to the algorithm. There are 4012 nodes in total, as shown in Figure 2. 


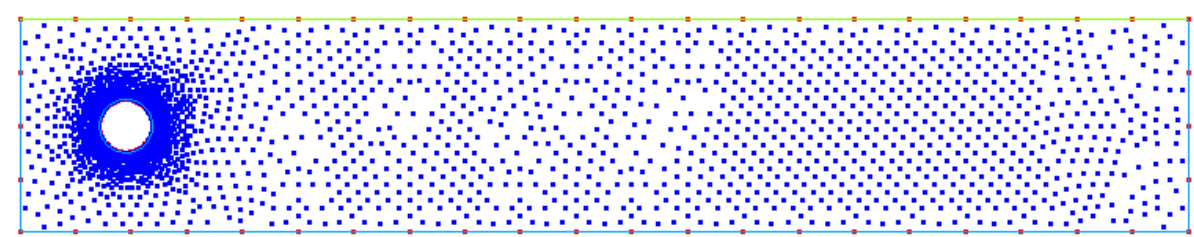

Figure 2. Automatic nodes arrangement in calculated area

In order to improve the calculated accuracy, we partly concentrate the nodes in the wake area (select a smaller $r$ value), according to the character of VIV wake on the basis of Figure 2. So, there are actually 5922 nodes in total. Nodes are shown in Figure 3.

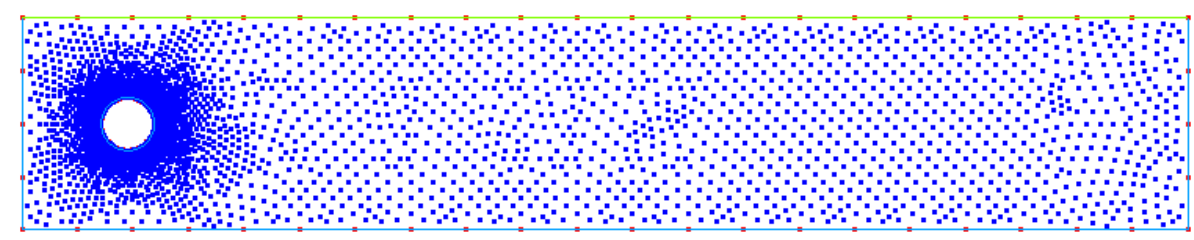

Figure 3. Automatic nodes arrangement in calculated area

\subsection{Calculated result and analysis}

According to the nodes arrangement above, calculate the vortex shedding shape when $\mathrm{Re}=100,200$ and 1000 , as shown in Figure 4.

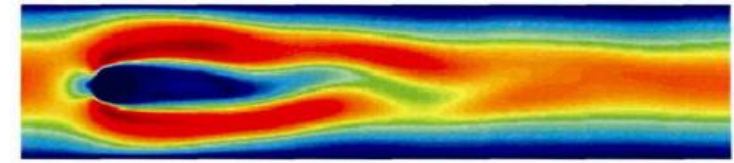

(a) $\operatorname{Re}=100$

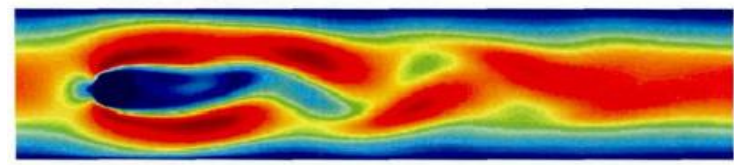

(b) $\operatorname{Re}=200$

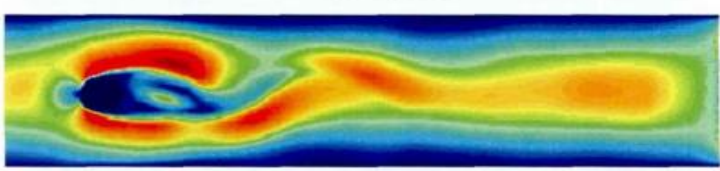

(c) $\mathrm{Re}=1000$

Figure 4 . The vortex shedding form of automatic nodes arrangement

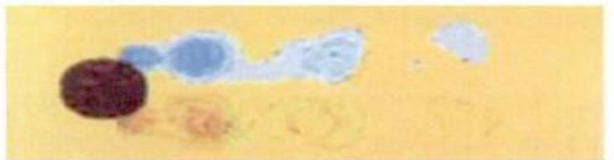

(a)

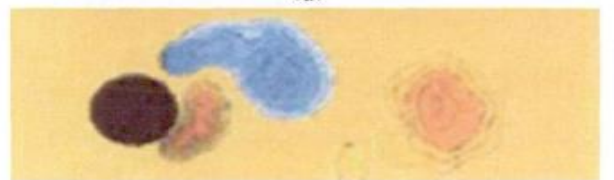

(b)

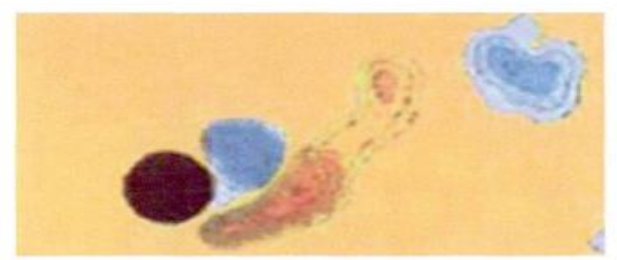

(c)

Figure 5. Flow field shape obtained by physical model experiments 
From Figure 4, we can conclude that the method mentioned can simulate the shape of vortex alternate shedding in the cylinder wake (Carmen Vortex Street) well. When $\operatorname{Re}$ is small ( $\operatorname{Re}=20, t=100 \mathrm{~s}$ ), flow field is symmetrical to the vortex in the back of cylinder, and the flow field is steady. With Re gradually increasing, the vortex becomes asymmetrical. So, when Re exceeds a certain threshold, it becomes instable and starts shedding from the cylinder, which evolves into regular vortex shedding. Figure 5 is the vortex shape obtained by a physical model experiment [6,7]. From comparison, the calculated results coincide well with experiment result, which can simulate the vortex shapes in different Re.

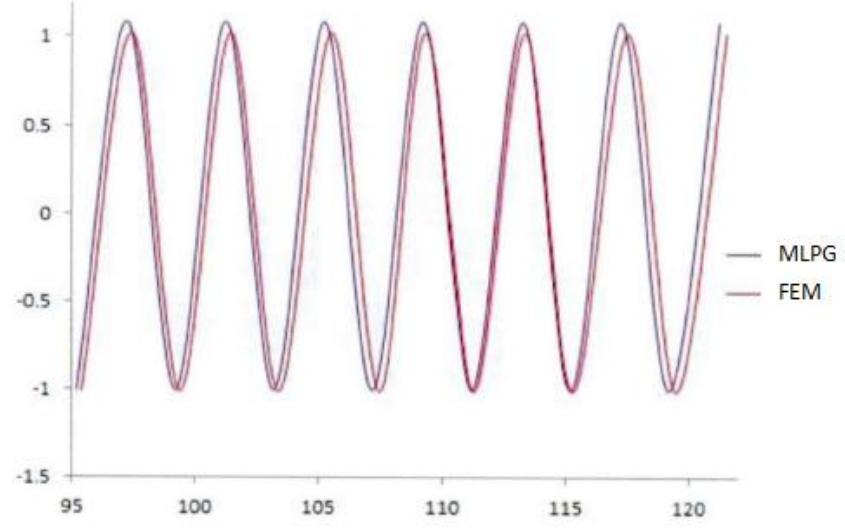

(a)The lift coefficient

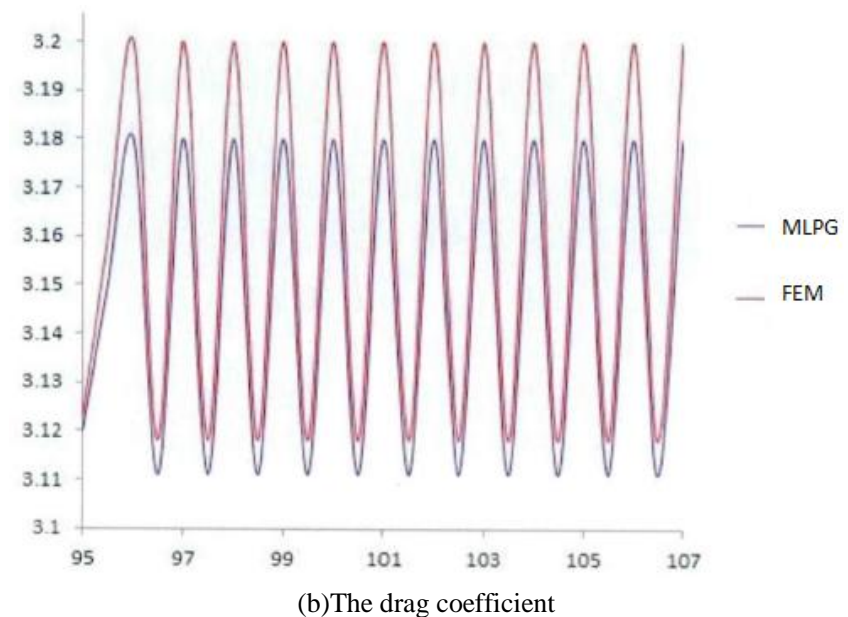

Figure 6. The lift and drag coefficient obtained from different nodes arrangements

\begin{tabular}{|c|c|c|c|c|}
\multicolumn{6}{|c|}{ Table 1. The results of lift and drag coefficient (Re=100) } \\
\hline & $C_{l, \min }$ & $C_{l, \max }$ & $C_{d, \min }$ & $C_{d, \max }$ \\
\hline FEM & -1.012 & 1.023 & 3.119 & 3.201 \\
\hline MLPG & -1.021 & 0.987 & 3.165 & 3.228 \\
\hline
\end{tabular}

To further analyze and compare the validity of the mentioned method, we calculate lift coefficient $C_{l}$ and drag coefficient $C_{d}$ respectively by the mentioned method and the traditional FEM method when $\operatorname{Re}=100$.

$$
\left\{\begin{array}{l}
C_{d}=\frac{f_{x}}{\rho U^{2} / 2} \\
C_{l}=\frac{f_{y}}{\rho U^{2} / 2}
\end{array}\right.
$$


Where $f_{x}$ and $f_{y}$ are respectively forces the cylinder subjected to in different direction $x$ and direction $y$, which can be obtained by integrating the pressure and viscous force subjected to the surface of the cylinder as Equation (15).

According to Equation (15), lift coefficient $C_{l}$ and drag coefficient $C_{d}$ can be obtained. Figure 6 is the lift and drag coefficient obtained from the two calculating method. Table 1 is the reference value comparison.

As shown with Figure 6 and Table 1, the calculated results conducted by the mentioned method and the traditional FEM method coincide well. To further analyze the validity and accuracy of the mentioned method, we can testify them by calculating the Strouhal number $S t$ of the oscillation cycle characterization lift coefficient $C_{l}$. It is shown in Figure16.

$$
S t=\frac{D}{U T}
$$

Where $T$ is the oscillation cycle as Equation (16).

The calculated results are shown in Table 2 and compared with other numerical values obtained by the physical model experiment and other numerical simulation method [12].

Table 2. The results of Strouhal number with different Res

\begin{tabular}{|c|c|c|c|c|}
\hline \multirow{2}{*}{} & \multicolumn{2}{|c|}{ St } & \multirow{2}{*}{ Method } \\
\cline { 2 - 5 } & $\mathrm{Re}=100$ & $\mathrm{Re}=200$ & $\mathrm{Re}=1000$ & \multirow{2}{*}{ MLPG } \\
\hline Method with Paper presented & 0.170 & 0.197 & 0.240 & FEM \\
\hline Herfjord, 1995 & 0.168 & 0.196 & 0.234 & FVM \\
\hline Rengel and Sphaier, 1999 & 0.173 & 0.203 & 0.225 & Model Experiment \\
\hline Norberg, 2003 & 0.164 & 0.184 & 0.210 & \\
\hline
\end{tabular}

From Table 2, the results conducted by the method proposed in the paper coincide well with those by the traditional FEM, FVM method in different Re, which shows its good validity.

\section{Conclusions}

The paper presents numerical simulation technology of deep-water risers' fluid-solid interaction based on mesh-free MLPG method, which takes the stationary cylinder as an example to calculate, analyze and verify. It is a good agreement that the method proposed in the paper coincides well with those by the traditional mesh simulation method and physical model experiment, which prove that the calculated method for typical FSI problem in this paper (2D VIV analyze) is available. Compared with traditional finite element method, meshless method has an apparent advantage in calculating deep-water risers. When processing deep-water nonlinear large deformation problem, units of lower quality can be avoided because no meshing is required. When the structure changes, no re-meshing is required, which is beneficial to the optimization design of the structure. After analysis and comparison, the meshless MLFG interaction algorithm is effective for two-dimensional fluid-structure interaction analysis.

\section{Acknowledgements}

This work is partially supported by Guangdong Natural Science Foundation under Project (Project No: 2014A030313792 and No.: 2016A030313125), and Guangdong Traffic Science and Technology under Project (Project No: 2015-02-050). It is also supported by China Postdoctoral Science Foundation (Project No: 2015M581622).

\section{References}

1. T. Belytschko, P. Kpysl, Y. Krongauz, “A Three-Dimensional Explicit Element-Free Galerkin Method”, Int. J, Numer. Meth. Fluids, vol.21, no.2, pp.1253-1270,2014

2. W. CHEN. Z. ZHENG, "Vortex-Induced Vibration of Deepwater Flexible Riser Experiencing Internal-Wave-Induced Shear Flow”, Engineering Mechanics, vol.28, no.1212, pp.250-256,2011

3. C. Do, "An Element-Free Galerkin Method for Simulation of Stationary Two-Dimensional Shallow Water Flows in Rivers", Methods Appl. Mech. Engrg, vol.182, no.1, pp.89-107.2011 
4. R. Govardhan, C. Williamson, "Modes of Vortex Formation and Frequency Response for a Freely Vibrating Cylinder", Journal of Fluid Mechanics, vol.420, pp.85-130,2010

5. R. Govardhan, C. Williamson, "Resonance Forever: Existence of a Critical Mass and an Infinite Regime of Synchronization in Vortex Induced Vibration", Journal of Fluid Mechanics, vol. 473, pp.147-166,2002

6. N. Jauvtis. and C. Williamson, "Vortex-Induced Vibration of a Cylinder with Two Degrees Off Freedom", Journal of Fluids and Structures, vol.17, pp.1035-1042,2013

7. N. Jauvtis. and C. Williamson, "The Effect of Two Degrees of Freedom on Vortex-Induced Vibration at Low Mass and Damping", Journal of Fluid Mechanics, vol.509, pp.23-62,2011

8. A. Khalak, C. Williamson, "Dynamics of a Hydro Elastic Cylinder with Very Low Mass and Damping", Journal of Fluids and Structures, vol.10, pp.455-472,1996

9. A. Khalak, C. Williamson, "Motions Forces and Mode Transitions in Vortex Induced Vibrations at Low Mass-Damping", Journal of Fluids and Structures, vol.13, pp.813-851,1999

10. S. Lei, W, Zhang, "Influence of Heave Motion on Vortex-Induced Vibrations of FDPSO Risers", Engineering Mechanics, vol.27, no.1, pp.294-298,2010

11. J. Liu, W. Huang, "A Nonlinear Vortex Induced Vibration Model of Marine Risers", Journal of Ocean University of China, vol.12, no.1, pp.32-36,2013

12. C. Norberg, "Fluctuating Lift on a Circular Cylinder: Review and New measurements", Journal of Fluids and Structures, vol.17, pp.57-96,2003

13. L. Singh, P. Jain, "Parallel EFG Algorithm for Heat Transfer Problems", Advances in Engineering Software, vol.36, no.8, pp.554-560,2005

14. Y. Sun, M. Li, "Nonlinear Approach of Vortex-Induced Vibration for Line-Like Structures", J. Wind Eng. Ind. Aerodyn, vol.124, pp. $1-6,2014$

15. P. Wang, H. Xue, "In-Line VIV Response and Fatigue Damage of a Deepwater Riser in Linearly Sheared Flow", Journal of Vibration and Shock, vol.32, no.19, pp.1-6,2013

16. Y. Wang, W. CuiI, "Effects of Various Factors on The VIV- Induced Fatigue Damage in Deep Sea Risers", Journal of Ship Mechanics, vol.10, no.5, pp.76-83,2006

17. Y. Wu, W. Huang, "An Analysis Method for Deepwater TLR Coupled Vibration of Parameter Vibration and Vortex-Induced Vibration", Applied Mechanics and Material, vol.284, pp.437-441,2013

18. H. Yang, H. Li, "Instability Assessment of Deep-Sea Risers Under Parametric Excitation", China Ocean Engineering, vol.23, no.4, pp.603-612,2009

19. X. Zhang, H. Xue, "VIV Fatigue Reliability and Fatigue Safety Factor Analysis of Deep-Water Riser", The Ocean Engineering, vol.32, no.6, pp.17-23,2014 